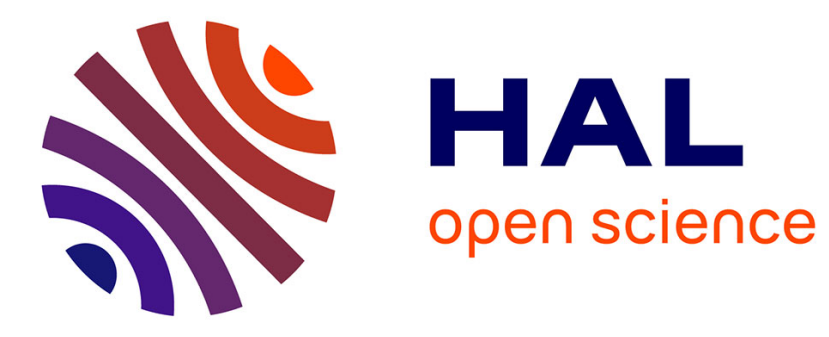

\title{
Systems Biology, Systems Medicine, Systems Pharmacology: The What and The Why
}

Angélique Stéphanou, Eric Fanchon, Pasquale Innominato, Annabelle Ballesta

\section{To cite this version:}

Angélique Stéphanou, Eric Fanchon, Pasquale Innominato, Annabelle Ballesta. Systems Biology, Systems Medicine, Systems Pharmacology: The What and The Why. Acta Biotheoretica, 2018, 66 (4), pp.345-365. 10.1007/s10441-018-9330-2 . hal-01975961

\section{HAL Id: hal-01975961 \\ https://hal.science/hal-01975961}

Submitted on 30 Sep 2021

HAL is a multi-disciplinary open access archive for the deposit and dissemination of scientific research documents, whether they are published or not. The documents may come from teaching and research institutions in France or abroad, or from public or private research centers.
L'archive ouverte pluridisciplinaire HAL, est destinée au dépôt et à la diffusion de documents scientifiques de niveau recherche, publiés ou non, émanant des établissements d'enseignement et de recherche français ou étrangers, des laboratoires publics ou privés. 


\title{
Systems Biology, Systems Medicine, Systems Pharmacology: The What and The Why
}

\author{
Angélique Stéphanou $^{1}$. Eric Fanchon ${ }^{2}$. \\ Pasquale F. Innominato ${ }^{3,4,5}$. Annabelle \\ Ballesta $^{4,5}$
}

Received: date / Accepted: date

\begin{abstract}
Systems biology is today such a widespread discipline that it becomes difficult to propose a clear definition of what it really is. For some, it remains restricted to the genomic field. For many, it designates the integrated approach or the corpus of computational methods employed to handle the vast amount of biological or medical data and investigate the complexity of the living. Although defining systems biology might be difficult, on the other hand its purpose is clear: systems biology, with its emerging subfields systems medicine and systems pharmacology, clearly aims at making sense of complex observations/experimental and clinical datasets to improve our understanding of diseases and their treatments without putting aside the context in which they appear and develop. In this short review, we aim to specifically focus on these new subfields with the new theoretical tools and approaches that were developed in the context of cancer. Systems pharmacology and medicine now give hope for major improvements in cancer therapy, making personalized medicine closer to reality. As we will see, the current challenge is to be able
\end{abstract}

\footnotetext{
1 Université Grenoble Alpes, CNRS, TIMC-IMAG/DyCTIM2 38000 Grenoble, France

${ }^{2}$ Université Grenoble Alpes, CNRS, TIMC-IMAG/BCM 38000 Grenoble, France

3 North Wales Cancer Centre, Betsi Cadwaladr University Health Board Denbighshire, United Kingdom

4 INSERM and Université Paris 11 Unit 935

Villejuif, France

${ }^{5}$ University of Warwick

Coventry, United Kingdom
} 
to improve the clinical practice according to the paradigm shift of systems sciences.

Keywords big data $\cdot$ precision medicine $\cdot$ personalized medicine $\cdot$ drug development $\cdot$ multi-scale approach

\section{Introduction}

Systems biology aims at considering biological problems in an integrated way. It is commonly opposed to the reductionist view on the principle that interactions between biological entities are as important as the properties of the entities themselves which taken alone are insufficient to describe the system's behaviour. A striking example was the gene-centred view of life according to which genes alone contained all the necessary information to explain the features, and hence, behaviours of all living entities including the emergence and development of diseases. The race for genome sequencing was then bearing the hopes to at last identify the roots of major diseases such as cancer but these expectations were never met. Following these disillusions, it became clear that the answers do not lie only in genes. Systems biology, that emerged at the beginning of the new millennium from the human genome project itself, followed the development of bioinformatics as a mean to process the huge amount of genomic data produced. To make sense of the data, the relationships between genes, proteins and their function in signalling and metabolism were identified and interpreted in biological networks, thus marking the transition from bioinformatics (data processing and statistical analysis) to systems biology (data integration). Although genomics, proteomics, metabolomics and other -omics remain at the heart of systems biology (Fig. 1), it has developed much beyond these fields making it now more difficult to define. The paradigm shift to think biology beyond the genome became more obvious as pointed out in the recent special issue "From the century of the genome to the century of the organism: New theoretical approaches" [83].

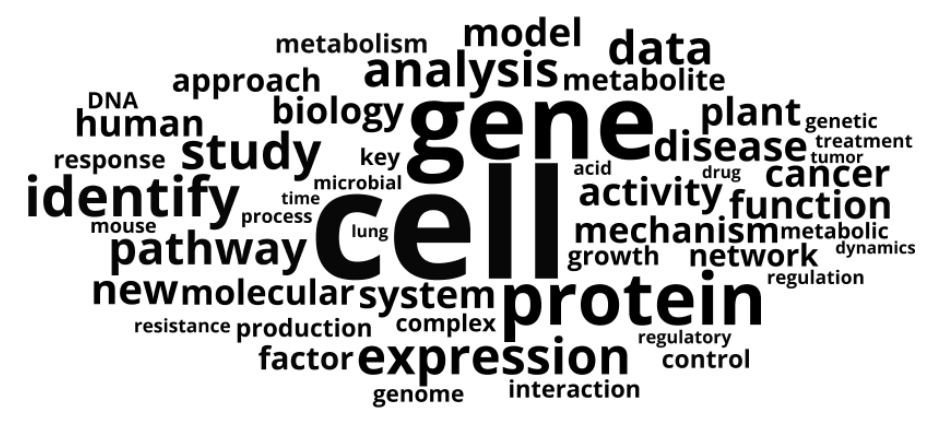

Fig. 1 Cloud of the 50 most cited words from paper abstracts extracted in Pubmed with research term "systems biology". Cell, gene and protein remain at the heart of this field. 
There is no universally acknowledged definition for systems biology [62], and is systems biology truly holistic? The term Holism has a variety of acceptations and is generally opposed to Reductionism. The first occurrence of the term in English is in J C Smuts' book Holism and Evolution [80]. In Smuts' own words, "Holism is a process of creative synthesis", and "it is the very essence of the concept of the whole that the parts are together in a combination, in a specific internal relatedness, in a creative synthesis which differentiates it from all other forms of combination or togetherness." It is claimed that some properties of a given system cannot be explained or predicted by its constituting parts and their interactions alone (although some other properties can).

These ideas are controversial and discussing this issue goes beyond the purpose of this manuscript. Let us just point out that the practice of Systems Biology, Medicine and Pharmacology (SBMP), as we will see it in this review, does not match the conception summarized above. In SBMP the system is defined by its constituents and their interactions (plus the interactions with the environment) and all the system properties are assumed to derive from them. There is no need to invoke a mysterious "creative synthesis" in this approach.

In SBMP the emphasis is on the System as opposed to classical molecular biology which focused on individual genes and proteins. But conceptually, this is close to what physicists do. The representation of the world given by Physics is stratified into levels (elementary particles, nucleus, atoms, molecules, etc.). Each level is associated to a subfield of physics, with its own set of concepts and laws, and one of the goals of physical theory is to understand how these entities and laws emerge from the lower level. The situation is identical in Systems Biology: understand how dynamical properties of cells emerge from the web of interactions at the molecular level. This is expressed explicitly in Pollard, 2003 [71]: "The reductionist tasks include an inventory of the relevant molecules, determination of molecular structures, identification of molecular partners, measurement of rate and equilibrium constants for each reaction, localization of the molecules in live cells, physiological tests for participation in cellular processes and formulation of mathematical models to understand the system's behaviour."

That said, one should keep in mind that cells contribute to build their (micro)environment. For example cells embedded in a tissue have an influence on the mechanical properties of the extracellular matrix by synthesizing fibers or by secreting proteases which cut fibers (ECM remodelling). In the other direction (from higher level to cell level) mechanosensitive molecules and cellular components, such as integrins and cytoskeletal filaments, have been shown to contribute to mechanotransduction pathways, i.e. signalling pathways by which cells convert mechanical signals into a biochemical response. In that way mechanical changes in the cellular microenvironment (tissue level) may influence cellular behaviour [29,37]. In this example there is thus a bidirectional coupling between two levels of organization, cell and tissue levels. This coupling also involves different time scales, the cells at a given time residing in a tissue produced at previous times. The modelling of such system on long 
time scales would requires multi-scale approach. To gain insight one must integrate various data types, integrate levels of organization, integrate organs to reason on the whole body. We could therefore argue that SBMP is in fact reductionist and integrative.

The Pioneer 100 Wellness Project (P100) [73] is a prominent early example of Systems Medicine. The study involved several omics technologies and activity measurements using wearable devices to generate a data cloud associated to each participant. The authors use the term 'holistic approach', so we would like to make another comment on its use. The meaning here is presumably different from the one presented above. It seems to be related rather to the use of several data types of different natures (omics data, clinical tests, activity tracking) in an effort to encompass several dimensions of the life of an individual. Without entering the debate about the benefit of the approach, we would like to point out that there is in fact kind of a paradox to claim that it is holistic when the patient is reduced to a cloud of data points (the patient is reified). Vogt et al. (2016) [87] call techno-scientific holism this new "holism". There is an instrumental rationality at work which seems to reduce the patient as a subject. A patient is a human being with an individual life history and may not be reduced to a data cloud when her/his health is concerned. If holism can be called for, it is indeed here. The patient should be considered as a whole, with her/his life history, culture and emotional states, her/his family environment, work conditions, social status, etc. The biopsychosocial model $[27,18]$ proposes to integrate biological factors (genetic, biochemical, etc.), with psychological and social factors.

Problems are not all necessarily solved with new technological devices. A broad reflection must take place in order to make the most of the new technologies (omics, wearable devices, Artificial Intelligence, Internet of Things, ...)

Despite controversies the consensus to define systems biology remains on the concept of integration. Systems biology is now often assimilated to the corpus of methods that allow to probe complex systems, i.e. a system that cannot be understood based on the knowledge of its sub-parts alone. Theoretical modelling, including computational biology, is one such very efficient method to investigate these systems. Systems biology then naturally bears the idea of in silico modelling as a mean to test new hypotheses and to build coherent frameworks to elaborate new theories.

Systems biology is today hugely involved in cancer research and bears new hopes for deciphering the roots of this disease [82]. It also appears as a mean to achieve personalized medicine [62] following the rise of precision medicine (Fig. 2). Systems medicine and systems pharmacology recently emerged as new major subfields [8], which in the context of cancer, allow to consider both treatment efficacy and tolerability in individual patients in a longitudinal manner, thus accounting for the emergence of all types of acquired resistance.

In this short review, we mainly focus on the context of cancer. In section 2 , we will first present and describe the fast-rising fields of systems medicine and systems pharmacology. We will focus in section 3 on the new tools that 


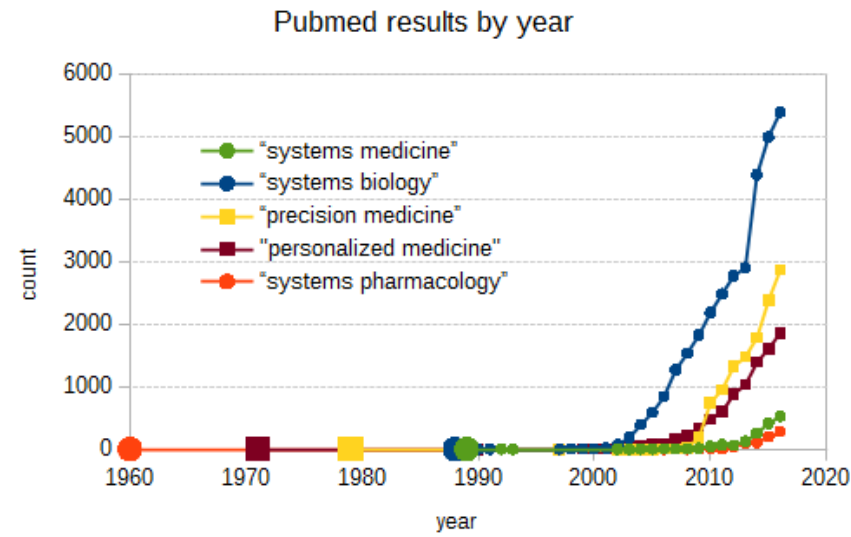

Fig. 2 First occurrence and rise of systems sciences and related precision/personalized medicine.

were developed to acquire and treat the data in these fields. The approaches and model types that now form the corpus of methods of systems biology will be described in section 4 . The impacts on research, formation and society will be discussed in section 5 .

\section{The emerging fields}

Systems Medicine: precision and personalized medicine

Systems medicine stems from the groundwork of systems biology and systems pharmacology, implementing and translating the more basic approaches to applied clinical research and practice. Systems medicine involves the implementation of such theoretical approaches in medical concepts, research and practice, through iterative and reciprocal feedback exchanges between clinicians, biologist, pharmacologists, bioinformaticians and mathematicians. Thus, systems medicine is indissociable from the other systems approaches, integrating at its very core reciprocal interplay with computational modelling of multidimensional sources of information. Accordingly, the driving force of ultimately improving patients outcomes goes through reiterated exchanges between bedside investigations, experimental models and computational analyses [14,21]. This has been emphasized in the Roadmap of the Coordinated Action for Systems Medicine (CaSyM) from the European Union (https://www.casym.eu) [22], and in other international consortia $[40,89,3,2,24,5]$. The final aim is a measurable improvement of patient health through systems-based approaches and practice [17]. One such instance of amelioration of standard of care through systems medicine is provided by the new classification of pediatric allergic diseases through an extensive translational approach spanning prediction, di- 
agnosis, prevention and therapeutics [19]. Another international effort resulting in beneficial changes in clinical practice involved chronic pulmonary diseases, whose temporal patterns can now be more accurately predicted and thus treated with the multidimensional systems approach [85]. Additionally, heterogeneous and challenging disorders such as irritable bowel disease have also beneficed from a systems-based modelling [58]. The fastest growing body of systems-driven data which will ultimately lead to improved treatment concerns nevertheless cancer, which is the paradigmatic example of heterogeneous and evolving pathology, whose morbidity, mortality and drug attrition rates are most defying for current medicine [28,78,44,54].

In complement to this integrated approach of systems biology and pharmacology applied to clinics, systems medicine encompasses the aspects of "anticipatory" and precision medicine, recapitulated in the $4 \mathrm{P}$ medicine conceptual framework $[81,34]$. The $4 \mathrm{Ps}$ stand for personalized, predictive, preventive and participatory medicine. This kind of rapidly emerging and expanding approach goes above and beyond the simple single-biomarker stratification, up to a concept of $\mathrm{N}=1$ study, where the intervention is fully tailored to the individual patient, disease and moment in time on the clinical evolution [90,61]. In order to be able to obtain such a degree of accuracy and "precision", multiple data sources are required, whose model-based integration provides a systemic and dynamical clinical picture, ultimately leading to the identification of the optimal intervention in terms of benefit and safety [49,30]. Figure 3 displays the conceptual outlook of the main innovative features of systems medicine which ought to drastically improve the clinical management of individuals.

\section{Systems Pharmacology}

The outcome of any therapeutic strategy may be largely affected by both patient- and disease-specific genetic or epigenetic alterations. For instance, cancer management is complicated by large inter-patient variabilities in both cancer molecular profiles and dynamics and drug response of healthy and tumour tissues. Consequently, treatment personalisation is required to ensure optimal therapeutic index. To this end, multi-type and multi-scale datasets are nowadays produced in cell cultures, in laboratory animals and in clinical investigations involving populations of patients or individual subjects. These large volumes of data which are thus generated across species require dedicated approaches to properly analyse each individual dataset, to handle the complexity arising from multiple data types and dimensions, and to finally translate the results into optimal therapies. Thus, systems pharmacology involves the use of biological, clinical, mathematical and computational means to critically improve drug development and pharmacotherapy personalization.

Systems pharmacology approaches are usually based on the host physiology and the molecular aspects of the disease. Hence, such investigations do not subdivide living organisms into independent components, but rather, recognise that genes, proteins, cells and organs interact with each other and 


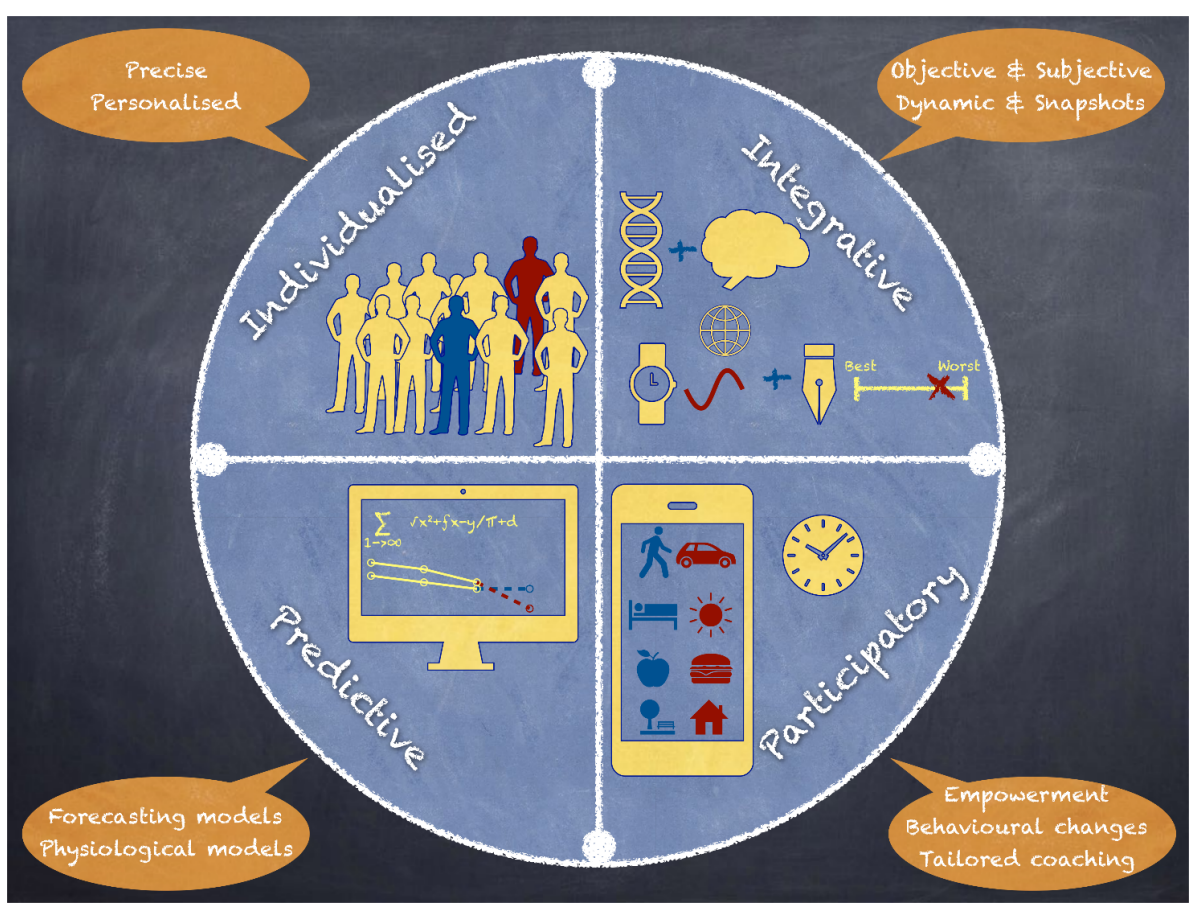

Fig. 3 Innovative hallmarks of Systems Medicine. It depicts the systems approach involving the four hallmarks (blue circle), the cross-disciplinary tools pertinent to each hallmark (yellow icons) and the features to be implemented to maximize the impact (orange clouds).

with the environment in complex ways that can vary over time. Further, the mathematical models aim to predict drug toxicity and efficacy which are ultimately determined by the response of gene and protein networks involved in the drug pharmacokinetics (PK) and pharmacodynamics (PD) in different cell populations - healthy or diseased - located in different organs. Hence, theoretical models of whole-body drug PK and cell type-specific regulatory pathways involved in drug PD constitute a reliable physiological basis from which the treatment can be optimized. Such detailed molecular and dynamical mathematical models further allow for the direct integration of the patients and diseased tissues molecular profiles into treatment decision. However, this complex physiology and its temporal organization are unlikely to be completely assessed in individual patients due to the invasive and potentially unethical nature of the clinical measurements that would be required. One strength of these physiologically-based models is the possibility to design human models though multi-scale pipelines [69]. Indeed, the mathematical models are based on the molecular physiology and their variables and parameters do have a physical meaning which is conserved across considered scales. Hence, sub-model structures and parameter values may be validated in in vitro and pre-clinical studies and further integrated in models for patient populations or individual patients. Overall, multi-scale systems pharmacology approaches integrate experimental 
results in cell culture, rodent and clinical investigations to ultimately design human subpopulation- or patient-specific drug combinations and/or schedules.

A recent systems pharmacology use case was provided by SymCyp Ltd (Certara, UK) for studying the impact of patient genotype on the PK-PD of Rosuvastatin, a drug that lower cholesterol levels [74]. A physiologically-based PK-PD model of the drug was designed incorporating both the drug PD in the liver as the main site of drug action. The study focused on patient polymorphisms in the organic anion-transporting polypeptide 1B1 (OATP1B1) which is involved in the cellular uptake of rosuvastatin. Clinical data showed that although the area under the plasma concentration-time curve was increased by more than $60 \%$ in patients presenting certain genotypes, the cholesterol synthesis response was unchanged in these same patients. The PK-PD model provided molecular insights into the whole-body drug fate which allowed to quantitatively explain this observed disconnect in between PK and PD patient datasets [74].

\section{The new tools}

"Big Data" and Domomedicine

The multi-scale integrative modelling underpinning systems approaches necessitate the acquisition, storage and complex analysis of data from multiple sources $[75,45]$. The various omics which can be dissected with extreme resolution [86], the possible multi-scale physiological models which can incorporate a broad range of parameters [60] and the vast array of human behaviour which can be monitored $[48,53,73,79]$ alongside the accurate forecasting of the individualized impact of pharmacological and non-pharmacological interventions over time [46,67], all require sound technological tools. Thus, the dense and dynamic data needed for precise modelling should be acquired with minimal intrusiveness into the subjects life. The rapid emergence of accurate biosensors, wearables and trackers, smart appliances and formal medical devices, all interweaved within the Internet of Things, can provide a plethora of biometric and behavioural data which can be remotely accessed and analysed $[23,4,31]$ and completed and expanded with other subjective data [12,50]. One of the challenges ahead is the optimal integration of these multi-source data, with the precise and personalized identification of relevant ones, to minimize the risk of undue sensitive and private information to be at risk of inappropriate access [76]. One great advantage of such inclusive multi-source evaluation of the health status of a patient is the dynamic integration of data across the 24-hour span, thus accounting for overlooked time frames (mainly night) in research and practice to date, especially in cancer [39]. Thus, the variations of human physiology and behaviour, spanning from the cellular till the whole organism level, across the 24 -h period can be monitored and predicted with a systems approach to optimize interventions, especially in cancer $[70,10,1]$. Another advantage of such approach is the possibility of maintaining the pa- 
tients within their home environment, while collecting the appropriate data for decision making. This domomedicine approach has the potential to improve healthcare system performance, healthcare professionals workload and patients wellbeing and outcomes [38]. A recent illustration of the efficiency of domomedicine is the study by Basch et al. (2017) [12] which demonstrated a 5-month increase in the survival of metastatic cancer patients by the sole use of a web-based platform tele transmitting patient-reported symptoms to clinicians [13]

Moreover, non-invasive recording of rest-activity rhythms over several consecutive days through wrist-actigraphy performed in colorectal cancer patients either before or during chemotherapy demonstrated that circadian rhythms disruption was associated with worse prognosis [10]. These domomedicine studies highlighted the predictive power of circadian rhythms monitoring in cancer patients initiating the development of several technological plateforms. For instance, the European Project InCASA (http://www.incasa-project.eu) monitored at home over an average duration of 58 days, 31 metastatic cancer patients on treatment. Individual data on self-measured body weight, self-rated symptoms, and circadian rest-activity rhythm recorded by a wristaccelerometer were tele-transmitted daily to a server via the Internet, using a dedicated platform. Further, the French PiCADo project [57] aimed to develop a multi-pathology telecommunicating platform integrating several lightweight and portable technologies made interoperable (sensor, collector, geolocation watch, digital tablet, digital pen collector, information systems, electronic health records). This technological development allowed for noninvasive and automatic collection of different markers of the patients biological rhythms and health status, that could be transmitted to the physicians.

\section{State-of-the-art Mathematical tools}

Apart from experimental and technological developments, new mathematical tools have been designed for systems medicine in the very last decades. First, physiologically-based models representing specific intracellular pathways and their drug response in single cells or in homogenous cell populations may be based on ordinary differential equations (ODEs). Genes and proteins of regulatory networks of interest such as the cell cycle or cell death through apoptosis are the model state variables whereas model parameters are rate constants [10]. Next, the drug PK-PD may be represented at the cellular or whole-body level by ODE-based models. Equations then compute: i) the concentrations of the parent drug and its metabolites over time resulting from biochemical events such as activation, detoxification, passive diffusion or active transport, ii) the drug activity on intracellular pathways of interest.

A typical system of ODE-equations thus allows to compute the time evolution of different quantities that depends on one another. It takes the form 
of coupled equations such as:

$$
\begin{aligned}
& \frac{d A}{d t}=\alpha-k_{1} A \\
& \frac{d B}{d t}=k_{1} A-k_{2} B
\end{aligned}
$$

Where $A$ and $B$ are the quantities of interest - called "state variables" - and can represent a variety of things, for example the concentrations of two proteins $A$ and $B$ that activate/inhibit one another; the concentration of two molecules where $A$ degrades or transforms into $B$; the concentrations of a drug in the compartments $A$ and $B$ following the drug use, uptake and degradation through the organism. Accordingly $\alpha$ represents the activation of the system as an external stimulus or initial inputs (administration or injection) that increases $A$. The coefficients $k$ are constants that governs the transition/transformation/transport rates between $A$ and $B$ or in/out of $A$ and/or $B$ : $-k_{1} A$ in the first equation is a loss for the quantity $A$ that is a gain for the quantity $B$ since this term is positive in the second equation. It typically traduces the transformation of $A$ into $B$ or activation/stimulation of $B$ by $A ;-k_{2} B$ is typically a decay/degradation/elimination term of $B$ or through $B$. Depending on the context the system of equations can become very complex if many interacting variables needs to be considered. Moreover the reaction/transfer rates are not always constant and can be represented with more or less complex functions. This means that most of the time these systems of equations do not have analytical solutions. The solutions can only be approximated numerically through the models simulations. However the low computational cost associated to those ODE-based models allow for their use in parameter estimation and therapeutic optimization procedures.

The above-mentioned ODE-based models are helpful for simulating shortterm cell dynamics or effect of therapies. For optimization over several days or weeks, it is required to model the cell divisions and death at the level of a heterogeneous cell population. Partial derivative equations (PDEs) structured in time $(t)$ and age $(a)$ of the cell in its current cell cycle phase are then often used $[9,15]$. The models may account for several cell types with that may differentiate from one to another [7]. For each cell population, the model account for cell cycle entry and progression through the different cell cycle phases, cell division, cell differentiation and cell death.

If we consider two cell populations $P_{1}$ and $P_{2}$ - as the cells progress in the cell cycle - with $P_{1}$ the cells in the initial phases $G_{1} S$ and $P_{2}$ the cells in the remaining phases $G_{2} M$, then the system of equations is given by:

$$
\begin{gathered}
\frac{\partial P_{1}}{\partial t}(t, a)+\frac{\partial P_{1}}{\partial a}(t, a)=f\left(t, P_{1}(t, a)\right) \\
\frac{\partial P_{2}}{\partial t}(t, a)+\frac{\partial P_{2}}{\partial a}(t, a)=g\left(t, P_{2}(t, a)\right)
\end{gathered}
$$

In that example case, when a cell in $P_{1}$ reaches its age limit then it is transferred into the $P_{2}$ population. Similarly when a cell in $P_{2}$ reaches the age 
corresponding to the cell cycle duration then the cell $P_{2}$ leads to two new cells in $P_{1}$, thus accounting for cell division at the end of the $M$-phase. The functions $f$ and $g$ are formulated so as to account adequately for cell progression, differentiation and death as required and might therefore involve other variables (i.e. other cell populations). PDE models can be connected to ODE-based cellular models through $i$ ) transition functions from one cell cycle phase to another- representing the control of the circadian clock on the cell cycle [26], or the cell cycle arrest upon drug exposure [15] ii) cell death rates thus accounting for drug-induced apoptosis. Apart from being based on the physiology, these PDE-based models also present the advantage to have a low computational cost which is a prerequisite for being utilized in parameter estimation and therapeutic optimization procedures.

PDEs are also used to integrate spatiality in the models. In some circumstances, it can be useful to describe the drug distribution in the tumour tissue since it can be highly heterogeneous due to $i$ ) variations in the drug diffusion itself that depends on the cells density (tissue compaction) and to ii) local drug uptake by tumour cells driven by the expression of membrane transporters which may vary according to cell types (different clones) and cell states (cell cycle or circadian phases).

The partial differential equation used to follow the evolution in space $(\bar{x}$ is the space vector) and time $(t)$ of a diffusive drug of concentration $C(\bar{x}, t)$ takes the following form:

$$
\frac{\partial C}{\partial t}=D \nabla^{2} C+\alpha-\beta C-\gamma n C
$$

Where $\nabla^{2}$ is the Laplacian operator (space variations); $D$ is the diffusion coefficient of the drug; $\alpha$ is a source term (drug administration), here represented as a constant, but it can take a variety of forms and can depend on other parameters or variables; $-\beta C$ accounts for a spontaneous decay; $-\gamma n C$ is the uptake by the targeted cells with $n(\bar{x}, t)$ their concentration.

To represent this huge variability in cells (states, clones, etc.), it has become common to use an agent-based representation for the cells to account for the individual properties of each single cell, thus providing an explicit description of the cell evolution such as progression in its cycle, state transitions or mutations $[20,72]$. This means that the cell concentration $n(\bar{x}, t)$ described above is replaced by $n_{k}$ which represents the individual cell $k$ at one specific location. This cell is given individual properties in terms of migrating ability, cell cycle duration, uptake rate of nutrients or drugs, etc. Integrating both the drug heterogeneous spatial distribution through PDEs and the tumour cells through a multi-agent representation requires to interface two model types of different mathematical nature, the first being continuous, the second often discrete. This led to the development of hybrid model formulations that allows to transfer information between the two sub-models [84]. More generally, hybrid approaches became very useful to combine many different model types: 
- continuous/discrete, i.e. consideration of a cell population - a tumour mass for example - while distinguishing individual cells properties (to differentiate sub-clones);

- fast/slow, i.e. integration of different temporal scales such as the diffusion of a drug (seconds) and its long term effect (up to days);

- microscopic/macroscopic, i.e. integration of different spatial scale from cells interactions resulting in overall tissue organization;

- stochastic/deterministic, most cell events - like cell division or apoptosis for example - occur with some degree of variability from one cell to another. Those events can either be represented using probabilities (stochasticity) or deterministic rules (is some conditions are reached). Computing stochastic events has a higher cost than computing deterministic events, so depending on the context it might be more efficient and sufficient to implement deterministic rules for some of those events.

These are only a few model types examples. Those different model types involve a range of formalisms such as boolean networks, ODEs, PDEs, cellular automaton, multi-agents, etc. These approaches are fast developing in the specific context of systems biology since they provide tools to integrate the many heterogeneous components of the systems in order to study it as a whole.

For example the virtual heart developed by Denis Noble is integrating many different aspects including elements of cell metabolism, electro-physiology and tissue anatomy to account for wave front spreading and the reconstruction of the heart beat phases [66]. In a similar way, modelling tumour growth requires to integrate the tumour environment which is now known to play a crucial role in the tumour development [20]. It is characterized not only by its chemical nature (growth factors, enzymes, etc.) but also by its mechanical nature as a dense network of deformable fibers. This requires highly integrated model formulations that couple the chemistry to the mechanics [52].

\section{The theoretical approaches}

\subsection{Multi-scale approaches}

\section{Explicit spatio-temporal multi-scale}

"Multi-scale" can designates two different approaches. The first is to explicitly consider different metric scales temporally or spatially. For the temporal multi-scale case, fast events like chemical reactions, that occur in terms of microseconds or faster, can produce some effects on the system that can only be observed and measured at a long time scale, in terms of hours or even days. To deal with this specific multi-temporality, the standard strategy is to compute events from fast to slow while ensuring that the absolute time (or reference time) and time sequence of events are respected. On the spatial side, it is common to integrate events occurring from the sub-cellular scale (nano to micro-scale) to the tissue scale (millimetre scale), where the cell is often 
the hub for integration [84]. More precisely, intracellular molecular/genetic networks will define cells reactions/sensitivity to extracellular signals (drug diffusion field for example). Each mechanism is computed at its own specific scale and output/input variables of interest are identified and transmitted through the scales. Transferred data can be transmitted as is or might require to be transformed, processed or converted to make it usable at a different scale. Typically, interpolations can be used for downwards conversion of data and reciprocally calculation of a mean can be used for an upwards conversion.

\section{Functional multi-scale}

The second multi-scale approach does not consider the scales in terms of metrics but in terms of functional units. This approach is also often referred to as multi-level. It is usually used to design physiological models for humans which incorporates molecular details of the studied physiology and/or of the pharmacology of drugs of interest. Such models involve many kinetic parameters, so that the main challenge does not necessarily lay in achieving a close fit to the data which is facilitated by the large number of degrees of freedom inherent to the models, but rather in computing realistic values of parameters though careful estimation procedures. Indeed, those numerous parameters are unlikely to be directly measurable in individual patients so that a multi-scale approach is required for the design of clinically-relevant models. In physiologically-based models, parameters do have a physical meaning that can be independently evaluated in preclinical studies and subsequently scaled to humans. Thus, in order to arrive at the final patient-specific model, a pipeline of in vitro, in vivo and clinical investigations is needed. In vitro investigations allow the design of detailed models at the cellular level and mouse studies serve to design representations at the whole organism. Next, a model for average men and women cancer patient can be obtained by keeping the structure of the mouse model and resizing the parameters for humans. Mathematical models which are not based on the physiology only allow for allometric animal-to-human scaling typically using body weight or body surface area. These methods have been proven inaccurate in part because they do not consider the species-specific metabolic rates and expression of transporters and intracellular proteins. Current trends lay into the development of mechanistic approaches such as physiologicallybased scaling, which are promising procedures to integrate preclinical data into clinical modelling, yet they are still under development [6,22]. As an example, model parameters can be scaled from mouse to human as follows: $i$ ) sex-specific organ volumes can be inferred from literature for each species [25, 59], ii) intracellular reaction rates are usually kept unchanged from the preclinical models, iii) protein activities can be proportionally scaled according to inter-species in vitro studies, iv) blood/organ transport and drug clearance parameters are intended to be scaled using physiological information although such methods are still under development [11,16,42,47].

Such multi-scale approach was undertaken to design of a physiologicallybased model of temozolomide (TMZ) brain disposition in humans [11]. The 
study pipeline included PK investigations in buffer solutions, cell culture, mice, and patients. The pH-dependent conversion of TMZ into 5-(3-methyltriazen-1yl)imidazole-4-carboxamide(MTIC) and MTIC subsequent fragmentation into 4-amino-5-imidazole-carboxamide (AIC) and a methyldiazonium cation - the active species - were represented in a model integrating experimental PK results in buffer solutions. Next, TMZ cellular PK was represented through a two-compartment model incorporating the buffer solution model in both the extra- and intracellular medium. Model parameters were estimated from TMZ, MTIC, and AIC concentrations measured in U87 glioma cells. Next, a model of TMZ brain disposition in mice was developed and incorporated the cellular model of TMZ PK. The extra- and intracellular compartments of the latter now correspond to the interstitial fluid and tumour cells within the brain tumour. tumour cell membrane transport parameters were scaled from the in vitro model proportionally to volumes, and all intracellular parameters were inferred from the cellular study. Finally, the human model was obtained by keeping the mouse model structure and intracellular parameter estimates and scaling all transport parameters according to volumes. However, this naive model calibration led to an overestimation of observed TMZ concentrations in the brain interstitial fluid of cancer patients by up to fivefold.

\subsection{Data-driven and model-driven approaches}

Different systems investigations exist which differ by the scale of the produced information. Two main types of such studies can be distinguished: i) datadriven approaches and ii) model-driven approaches.

\section{Data-driven approach}

The first is the most recent and is mainly associated to so-called omics technologies. The suffix -omics is used to refer to the study of large sets of biological molecules, or pieces of information, and are based on high throughput experimental methods. A large number of omics technologies have been developed in the last two decades, including genomics, genotyping, transcriptomics, proteomics, metabolomics, fluxomics, interactomics, epigenomics and many others (an ever expanding list...) Using such technologies, it is possible to characterize for example the set of proteins contained in a given cell type in given conditions (at least the soluble proteins), or identify the set of overor under-expressed genes by comparing two conditions. They are wide-ranging and shallow, in the sense that they do not provide a detailed mechanistic understanding of a specific phenomenon but rather give a broad view of a domain (set of expressed genes, set of proteins, set of protein-protein interactions, etc). High-throughput (omics) data often lead to lists (e.g. the proteome of a given cell type), maps (e.g. protein-DNA and protein-protein interaction maps) or statistical associations (e.g. mutation associated to a given pathology). Most of the data types produced are static. A data set is associated to a cell type and 
state in a given experimental setting. There is no kinetic or time-related content, except of course in the case of microarray time-series data (time-course gene expression data) and fluxomics (metabolic fluxes). Multi-omics datasets combine several data types, e.g. Williams et al., 2016 [88] combine quantitative proteomics with genomics, transcriptomics and metabolomics, but the information obtained is still static, namely statistical correlations between traits and genomic variations across a population. The multi-omics approach are also used to characterize intra-tumour heterogeneity [68,77]. However multi-omics approaches, by assembling different pieces of information at several time points and several disease relevant tissues, have the potential to uncover molecular processes involved in the development of diseases, and distinguish those that are causal from others which are consequences [33]. And of course the same can be said about the mechanisms of resistance to treatments. Mathematical and computational models being natural frameworks to integrate data (see next section), it is likely that data-driven and model-driven approaches will get closer in the near future and become more and more complementary.

A deeper understanding of disease processes should thus lead to better quality biomarkers, new drug targets, and better patient stratification. It seems however unlikely that wide-ranging omics data collection (e.g. whole genomes, whole proteomes etc.) will be appropriate at the bedside. After the research phase (discovery) it is more cost-effective to perform focused tests, maybe on a wider scale than presently practiced, using array technologies. Another issue is the integration of molecular-level omics data with data on other levels: clinical physiological, behavioral, psychological. A step in that direction is the Pioneer 100 Wellness Project [73] already mentioned above, but in that study the data are interpreted by coaches in an informal way. New methods and tools will be needed to integrate all the data in a systematic and automated way in order to take into account human and environmental variability and design personalized treatment on this basis.

\section{Model-driven approach}

The second category of systems approaches are broadly speaking based on data from dedicated experiments and aimed at elucidating the biological mechanisms at work in the phenomenon of interest. Such approaches are focused on the specific phenomenon studied, and deep, in the sense that they go deeply into deciphering the molecular networks. They are based on mechanistic models which can be expressed in a variety of formalisms (such as ODEs, PDEs, Thomas network, stochastic differential equations) allowing to simulate the time evolution of a system. With the rise of the Systems view in Biology the concept of network has taken center stage. A network can be used to describe a regulatory structure (e.g. Gene Regulatory Network) or describe flows within a system (e.g. metabolic networks). Mechanistic models are necessary to rationalize and make sense of the vast array of experimental data. Such models constitute a framework to assemble in a consistent manner data of various types. Models represent in a formal way a state of knowledge and hypotheses 
at a given moment in the discovery process, and can be used to generate new hypotheses and choose the most relevant and informative experiments to be performed next. The discovery process is thus an iteration of experimentation and modelling steps, producing improved models at each iteration of the cycle. The drawback of mechanistic models is that they contain many parameters the value of which must be determined in order to able to perform simulations. Molecular network models are particularly complex to build and parametrize. This is due to the fact that the data with kinetic contents are still scarce, as mentioned above. This might seem paradoxical considering the plethora of omics data in general. A big challenge today is still to build mechanistic models of molecular networks from high-throughput data but examples do exist in the context of systems medicine [56].

\section{Impacts on Health Stakeholders}

\section{On drug development and clinical trial design}

Clinical attrition is a major bottleneck to transfer drug candidates from bench to clinical practice. Only approximately $10 \%$ of all compounds reaching phase I will eventually obtain authorization by the US Food and Drug Administration (FDA). Failure rates are in the $30 \%$ range in phase I, in the $60 \%$ range in phase II, 30 to $40 \%$ in phase III, and around $10 \%$ at the FDA submission to approval stage [51]. Oncology has the highest failure rate with over $93 \%$ of selected compounds being eventually rejected. To help addressing this issue, the NIH hosted several workshops on quantitative systems approaches for drug development and conclusions were published in a white paper in 2011 [32]. This publication highlighted the need for developing systematic approaches investigating the origins of inter- and intra-subject variability in drug response at the single-cell, organ and patient level based on genomic, proteomic, metabolomics and environmental investigations. It further recommended developing multi-scale PK-PD mathematical models incorporating pharmacological mechanisms at the cell to the whole organism level. Seven years after the release of this white paper, significant research advancements in each subtopic have been made. Eight success stories are highlighted in the Avicenna Roadmap [6] from innovative trial adaptative design [36] to PBPK modelling enabling pharmacodynamic prediction [43]. In 2007, the Entelos biosimulation company (http://www.entelos.com/) predicted from in silico studies that rituximab would have greater efficacy than tumour necrosis factor (TNF) inhibitor in preventing bone erosion in patients with severe joint diseases. They showed through computational simulations that rituximab would more efficiently preserve joint structure and decrease the rate of cartilage degradation compared to anti-TNF molecules. These model predictions predated a revision to the UK National Institute for Health and Care Excellence (NICE) guidelines by several years. Indeed, in 2010, rituximab and other related compounds were recommended by NICE for the treatment of rheumatoid arthritis in case 
of failure of prior anti-TNF drugs [55]. However, despite these very positive successes, initiatives to implement all the above recommendations to tackle the challenge of improving patient stratification and define better clinical trial methodologies remain scarce and highly challenging, especially in Oncology. Yet, systems medicine holds the promise to dramatically improve clinical trial design and outcomes through the use of virtual patients and in silico models (Figure 4).

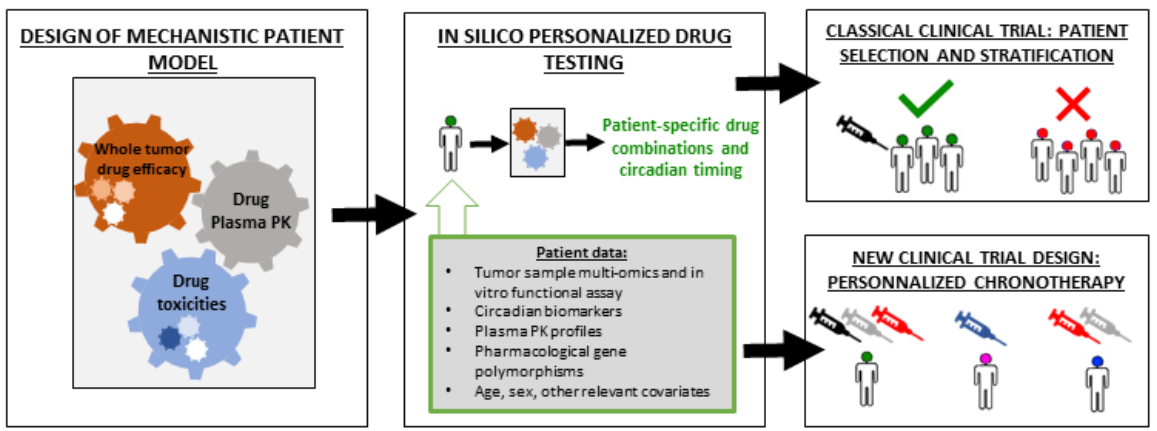

Fig. 4 In silico clinical trial for anticancer drug combinations.

On medical doctor, biologist and mathematician formation

The new concept of wellness rather than disease, pre-emptive rather than reactive intervention, individualized rather than aggregative approach, integrated rather than ultra-focussed evaluation, requires a profound paradigm-shift in the clinical management of a subject, as well as in the ideation of a research study. In order to achieve such transition, the training of the new generation of scientists and clinicians ought to incorporate the notions of systems approach, of cross-disciplinary and of integrative assessments. In pursuance of this goal, specific courses ought to be integrated within the learning curricula of all disciplines involved, biology and life sciences, mathematics, statistics, bioengineering, computational sciences, psychology and sociology, pharmacology and medicine. Such permeation should include both a basic conceptual introduction within the compulsory basic teaching, in order to broadly disseminate the systems framework, and more in-depth training courses, tailored to the individual disciplines, yet integrating the multi-disciplinary characteristic of the systems approach. Thus, a shared effort of the scientific community is to develop, update and incorporate these novel training sessions across the academic spectrum of curricula and schools. International societies and initiatives are indeed pursuing this ambitious goal. 


\section{Conclusions}

Back in 19th century, Claude Bernard had already realized the necessity and the huge potentiality of the systems approach. Denis Noble - following Bernard's insightful recommendations [64] - was the first to apply and prove the usefulness and efficiency of the multi-level system approach for the understanding of the heart $[35,63,66]$. This contributed to help in the identification of adapted drug treatment for a number of its pathologies [65]. Many medical applications of systems approaches have since been developed with success and some striking cases - such as AIDS modelling - can be found in the recent review of Joly and Rondó (2017)[41].

Systems investigations have all in common that they consist of a sequence of experimental and theoretical steps, in order to integrate the relevant components of a complex system in its different time and spatial scales. Such methodologies clearly enlarge the field of biological or medical questions that can now be addressed. In particular, they theoretically allow for precision and personalized medicine by the integration of multi-type datasets available in individual patients from blood tests to imaging, to self-reported symptom questionnaires.

However, despite very promising success stories, systems sciences have not yet been fully implemented in drug development, clinical trial design or the everyday clinical practice so far. This may be explained by the lack of proper formation for all actors of the field from biologists to clinicians, nurses, mathematicians and computer scientists. Indeed, such pluri-disciplinary projects require specific skills including the ability to work with professionals of various background.

Our future challenge lays thus in the design of new academic degrees fully integrating systems sciences at their core. The enrichment of existing professional careers with the ability to be part of pluri-disciplinary projects and the emergence of new jobs enabling the implementation of systems approaches in the clinics are key for the future development of the field.

\section{References}

1. Abdullah S., Murnane EL, Matthews M, Choudhury T. Circadian Computing: Sensing, Modeling, and Maintaining Biological Rhythms. Springer International Publishing AG 2017; 35 J.M. Rehg et al. (eds.), Mobile Health.

2. Agur Z, Elishmereni M, Kheifetz Y. Personalizing oncology treatments by predicting drug efficacy, side-effects, and improved therapy: mathematics, statistics, and their integration. Wiley Interdiscip Rev Syst Biol Med 2014;6:239-53

3. Anderson ARA, Quaranta V. Integrative mathematical oncology. Nat Rev Cancer 2008;8:227-34

4. Appelboom G, Camacho E, Abraham ME, Bruce SS, Dumont EL, Zacharia BE, D'Amico R, Slomian J, Reginster JY, Bruyère O, Connolly ES Jr. Smart wearable body sensors for patient self-assessment and monitoring. Arch Public Health 2014;72(1):28

5. Apweiler R, Beissbarth T, Berthold MR, Blüthgen N, Burmeister Y, Dammann O, Deutsch A, Feuerhake F, Franke A, Hasenauer J, Hoffmann S, Höfer T, Jansen PL, Kaderali L, Klingmüller U20, Koch I, Kohlbacher O, Kuepfer L, Lammert F, Maier D, Pfeifer 
N, Radde N, Rehm M, Roeder I, Saez-Rodriguez J, Sax U, Schmeck B, Schuppert A, Seilheimer B, Theis FJ, Vera J, Wolkenhauer O. Whither systems medicine? Exp Mol Med 2018;50(3):e453

6. Avicenna. March 2017. A strategy for in silico clinical trial. http://avicennaisct.org/about/

7. Avila JL, Bonnet C, Clairambault J, Ozbay H, Niculescu SI, Merhi F, Ballesta A, Tang R, Marie JP. Analysis of a new model of cell population dynamics in Acute Myeloid Leukemia. Advances in Delays and Dynamics, Delay Systems: Springer 2014;315-328

8. Ayers D, Day PJ. Systems Medicine: The Applications of Systems Biology Approaches for Modern Medical Research and Drug Development. Molecular Biology International 2015, ID698169.

9. Ballesta A, Clairambault J. Physiologically Based Mathematical Models to Optimize Therapies Against Metastatic Colorectal Cancer: a Mini-Review. Curr Pharm Des $2014 ; 20(1): 37-48$.

10. Ballesta A, Innominato PF, Dallmann R, Rand DA, Levi FA. Systems Chronotherapeutics. Pharmacological reviews 2017;69:161-99

11. Ballesta A, Zhou Q, Zhang X, Lv H, Gallo JM. Multiscale design of cell-typespecific pharmacokinetic/pharmacodynamic models for personalized medicine: application to temozolomide in brain tumors. CPT: pharmacometrics \& systems pharmacology 2014;3:e112

12. Basch E. Patient-Reported Outcomes - Harnessing Patients' Voices to Improve Clinical Care. N Engl J Med 2017;376:105-8

13. Basch E, Deal AM, Dueck AC, Scher HI, Kris MG, Hudis C, Schrag D. Overall Survival Results of a Trial Assessing Patient-Reported Outcomes for Symptom Monitoring During Routine Cancer Treatment. JAMA 2017;318(2):197-198

14. Benson M. Clinical implications of omics and systems medicine: focus on predictive and individualized treatment. J Intern Med 2016;279:229-40

15. Billy F, Clairambault J, Fercoq O, Gaubert S, Lepoutre T, Ouillon T, Saito S. Synchronisation and control of proliferation in cycling cell population models with age structure. Mathematics and Computers in Simulation 2014;96:66-94

16. Blanchard OL, Smoliga JM. Translating dosages from animal models to human clinical trials-revisiting body surface area scaling. Faseb j 2015;29:1629-34

17. Boissel JP, Auffray C, Noble D, Hood L, Boissel FH. Bridging Systems Medicine and Patient Needs. CPT: pharmacometrics \& systems pharmacology 2015;4:e00026

18. Borrell-Carrió F, Suchman AL, Epstein RM. The Biopsychosocial Model 25 Years Later: Principles, Practice, and Scientific Inquiry. Ann Fam Med 2004;2(6):576582

19. Bousquet J, Anto JM, Akdis M, Auffray C, Keil T, Momas I, Postma DS, Valenta R, Wickman M, Cambon-Thomsen A et al. Paving the way of systems biology and precision medicine in allergic diseases: the MeDALL success story: Mechanisms of the Development of ALLergy; EU FP7-CP-IP; Project No: 261357; 2010-2015. Allergy 2016;71(11):15131525

20. Caraguel F, Lesart AC, Estève F, van der Sanden B, Stéphanou A. Towards the design of a patient-specific virtual tumour. Comput Math Methods Med 2016;2016:7851789

21. Cascante M, de Atauri P, Gomez-Cabrero D, Wagner P, Centelles JJ, Marin S, Cano I, Velickovski F, Marin de Mas I, Maier D, Roca J, Sabatier P. Workforce preparation: the Biohealth computing model for Master and PhD students. J Transl Med 2014;12 Suppl 2:S11

22. THE CASyM ROADMAP Implementation of Systems Medicine across Europe. https://www.casym.eu/blog/publications/2017/the-casym-roadmap-updated-version-ofapril-2017-released/

23. Chung AE, Jensen RE, Basch EM. Leveraging Emerging Technologies and the "Internet of Things" to Improve the Quality of Cancer Care. Journal of oncology practice 2016;12:863-6

24. Collins FS, Varmus H. A new initiative on precision medicine. N Engl J Med 2015;372(9):793-795

25. Davies B, Morris T. Physiological parameters in laboratory animals and humans. Pharmaceutical research 1993;10:1093-5

26. El Cheikh R, Bernard S, El Khatib N. Modeling circadian clock-cell cycle interaction effects on cell population growth rates. J Theor Biol 2014;363:318-31 
27. Engel GL. The need for a new medical model: a challenge for biomedicine. Science 1977;196(4286):129-36

28. Filipp FV. Precision medicine driven by cancer systems biology. Cancer Metastasis Rev 2017;36(1):91-108

29. Geiger B, Bershadsky A, Pankov R, Yamada KM. Transmembrane crosstalk between the extracellular matrix-cytoskeleton crosstalk. Nat Rev Mol Cell Biol 2001;2(11):793-805

30. Gietzelt M, Lopprich M, Karmen C, Knaup P, Ganzinger M. Models and Data Sources Used in Systems Medicine. A Systematic Literature Review. Methods of information in medicine 2016;55:107-13

31. Gresham G, Schrack J, Gresham LM, Shinde AM, Hendifar AE, Tuli R, Rimel BJ, Figlin R, Meinert CL, Piantadosi S. Wearable activity monitors in oncology trials: Current use of an emerging technology. Contemp Clin Trials 2018;64:13-21

32. Group QW. 2011 Quantitative and Systems Pharmacology in the Post-genomic Era: New Approaches to Discovering Drugs and Understanding Therapeutic Mechanisms. An NIH White Paper. https://www.nigms.nih.gov/News/reports/Pages/201110syspharma.aspx

33. Hasin Y, Seldin M, Lusis A. Multi-omics approaches to disease. Genome Biol 2017;18(1):83

34. Hood L, Flores M. A personal view on systems medicine and the emergence of proactive P4 medicine: predictive, preventive, personalized and participatory. New biotechnology 2012;29:613-24

35. Hutter OF, Noble D. Rectifying properties of heart muscle. Nature 1960;188-495

36. Immunetrics. March 2017. Immunetrics Quantitative Systems Pharmacology (QSP) Modeling Services \& Technology: Designed in collaboration with modelers, for modelers. http://www.immunetrics.com.

37. Ingber DE. Cellular mechanotransduction: putting all the pieces together again. FASEB J 2006;20(7):811-27

38. Innominato PF, Komarzynski S, Mohammad-Djafari A, Arbaud A, Ulusakarya A, Bouchahda M, Haydar M, Bossevot-Desmaris R, Plessis V, Mocquery M, Bouchoucha D, Afshar M, Beau J, Karabou A, Morre JF, Fursse J, Rovira Simon J, Levi F. Clinical Relevance of the First Domomedicine Platform Securing Multidrug Chronotherapy Delivery in Metastatic Cancer Patients at Home: The inCASA European Project. J Med Internet Res 2016;18(11):e305

39. Innominato PF, Roche VP, Palesh OG, Ulusakarya A, Spiegel D, Lévi FA. The circadian timing system in clinical oncology. Ann Med 2014;46(4):191-207

40. Iyengar R, Altman RB, Troyanskya O, FitzGerald GA. MEDICINE. Personalization in practice. Science 2015;350:282-3

41. Joly M, Rondó PHC. The future of computational biomedicine: complex systems thinking. Math Comp Sim 2017;132:1-27

42. Kang HE, Lee MG. Approaches for predicting human pharmacokinetics using interspecies pharmacokinetic scaling. Archives of pharmacal research 2011;34:1779-88

43. Ke A, Barter Z, Rowland-Yeo K, Almond L. Towards a Best Practice Approach in PBPK Modeling: Case Example of Developing a Unified Efavirenz Model Accounting for Induction of CYPs 3A4 and 2B6. CPT: pharmacometrics \& systems pharmacology 2016;5:367-76

44. Kirchmair J, Göller AH, Lang D, Kunze J, Testa B, Wilson ID, Glen RC, Schneider G. Predicting drug metabolism: experiment and/or computation? Nat Rev Drug Discov 2015;14(6):387-404

45. Kirschner M. Systems Medicine: Sketching the Landscape. Methods Mol Biol 2016;1386:3-15

46. Kuepfer L, Schuppert A. Systems Medicine in Pharmaceutical Research and Development. Methods Mol Biol 2016;1386:87-104

47. Lave T, Chapman K, Goldsmith P, Rowland M. Human clearance prediction: shifting the paradigm. Expert opinion on drug metabolism \& toxicology 2009;5:1039-48

48. Li X, Dunn J, Salins D, Zhou G, Zhou W, Schussler-Fiorenza Rose SM, Perelman D, Colbert E, Runge R, Rego S, Sonecha R, Datta S, McLaughlin T, Snyder MP. Digital Health: Tracking Physiomes and Activity Using Wearable Biosensors Reveals Useful Health-Related Information. PLoS biology 2017;15(1):e2001402 
49. Liang Y, Kelemen A. Computational dynamic approaches for temporal omics data with applications to systems medicine. BioData mining 2017;10:20

50. Low CA, Dey AK, Ferreira D, Kamarck T, Sun W, Bae S, Doryab A. Estimation of Symptom Severity During Chemotherapy From Passively Sensed Data: Exploratory Study. J Med Internet Res 2017;19(12):e420

51. Lowe D. New Look At Clinical Attrition. Science translational medicine 2014; http://blogs.sciencemag.org/pipeline/archives/2014/01/10/a_new_look_at_clinical_attrition

52. Macklin P, Frieboes HB, Sparks JL, Ghaffarizadeh A, Friedman SH, Juarez EF, Jonckheere E, Mumenthaler SM. Progress towards computational 3-D multicellular systems biology. Adv Exp Med Biol 2016;936:225-46

53. Majumder S, Mondal T, Deen MJ. Wearable Sensors for Remote Health Monitoring. Sensors (Basel, Switzerland) 2017;17

54. Maley CC, Aktipis A, Graham TA, Sottoriva A, Boddy AM, Janiszewska M, Silva AS, et al. Classifying the evolutionary and ecological features of neoplasms. Nat Rev Cancer $2017 ; 17(10): 605-619$

55. Malottki K, Barton P, Tsourapas A, Uthman AO, Liu Z, Routh K, Connock M, Jobanputra P, Moore D, Fry-Smith A, Chen YF. Adalimumab, etanercept, infliximab, rituximab and abatacept for the treatment of rheumatoid arthritis after the failure of a tumour necrosis factor inhibitor: a systematic review and economic evaluation. Health Technol Assess $2011 ; 15(14): 1-278$.

56. Marin de Mas I, Fanchon E, Papp B, Kalko S, Roca J, Cascante M. Molecular mechanisms underlying COPD-muscle dysfunction unveiled through a systems medicine approach. Bioinformatics 2017;33(1):95-103

57. Maurice M, Lévi F, Breda G, Beaumatin N, Duclos A, Chkeir A, Hewson D, Duchêne J. Innovative project for domomedicine deployment, The PiCADo Pilot Project. eTELEMED 2015: The Seventh International Conference on eHealth, Telemedicine, and Social Medicine.

58. Mayer EA, Labus JS, Tillisch K, Cole SW, Baldi P. Towards a systems view of IBS. Nat Rev Gastroenterol Hepatol 2015;12(10):592-605

59. McConnell EL, Basit AW, Murdan S. Measurements of rat and mouse gastrointestinal $\mathrm{pH}$, fluid and lymphoid tissue, and implications for in-vivo experiments. J Pharm Pharmacol 2008;60:63-70

60. Mizeranschi A, Groen D, Borgdorff J, Hoekstra AG, Chopard B, Dubitzky W. Anatomy and Physiology of Multiscale Modeling and Simulation in Systems Medicine. Methods Mol Biol 2016;1386:375-404

61. Morere JF, Innominato P. ESMO 2014: new trends in precision medicine. Targeted oncology 2014;9:293-4

62. Naylor S, Chen JY. Unraveling human complexity and disease with systems biology and personalized medicine. Personalized medicine 2010;7:275-89

63. Noble D. Cardiac action and pacemaker potentials based on the Hodgkin-Huxley equations. Nature 1960;188:495-7

64. Noble D. Claude Bernard, the first systems biologist, and the future of physiology. Exp Physiol 2008;93(1):16-26

65. Noble D, Colatsky TJ. A return to rational drug discovery: computer-based model of cells, organs and systems in drug target identification. Expert Opin Ther Targets 2000;4:39-49

66. Noble D. From the Hodgkin-Huxley axon to the virtual heart. J Physiol 2007;580(1):1522

67. Orr MG, Plaut DC. Complex systems and health behavior change: insights from cognitive science. American journal of health behavior 2014;38:404-13

68. Ortega MA, Poirion O, Zhu X, Huang S Wolfgruber TK, Sebra R, Garmire LX. Using single-cell multiple omics approaches to resolve tumor heterogeneity. Clin Transl Med 2017;6:46

69. Ortiz-Tudela E, Mteyrek A, Ballesta A, Innominato PF, Levi F. Cancer chronotherapeutics: experimental, theoretical, and clinical aspects. Handb Exp Pharmacol 2013;217:26188

70. Ozturk N, Ozturk D, Kavakli IH, Okyar A. Molecular Aspects of Circadian Pharmacology and Relevance for Cancer Chronotherapy. Int J Mol Sci 2017;18(10). pii: E2168 
71. Pollard TD, The cytoskeleton, cellular motility and the reductionist agenda. Nature 2003;422:741-5

72. Powathil GG, Adamson DJ, Chaplain MA. Towards predicting the response of a solid tumour to chemotherapy and radiotherapy treatments: clinical insights from a computational model. PLoS Comput Biol 2013;9(7):e1003120

73. Price ND, Magis AT, Earls JC, Glusman G, Levy R, Lausted C, McDonald DT, Kusebauch U, Moss CL, Zhou Y, Qin S, Moritz RL, Brogaard K, Omenn GS, Lovejoy JC, Hood L. A wellness study of 108 individuals using personal, dense, dynamic data clouds. Nature biotechnology 2017;35:747-56

74. Rose RH, Neuhoff S, Abduljalil K, Chetty M, Rostami-Hodjegan A, Jamei M. Application of a Physiologically Based Pharmacokinetic Model to Predict OATP1B1-Related Variability in Pharmacodynamics of Rosuvastatin. CPT Pharmacometrics Syst Pharmacol 2014;3:e124.

75. Saqi M, Pellet J, Roznovat I, Mazein A, Ballereau S, De Meulder B, Auffray C. Systems Medicine: The Future of Medical Genomics, Healthcare, and Wellness. Methods Mol Biol 2016;1386:43-60

76. Serrano KJ, Yu M, Riley WT, Patel V, Hughes P, Marchesini K, Atienza AA. Willingness to Exchange Health Information via Mobile Devices: Findings From a PopulationBased Survey. Annals of family medicine 2016;14(1):34-40

77. Shi L, Zhang Y, Feng L, Wang L, Rong W, Wu F, Wu J, Zhang K, Cheng S. Multi-omics study revealing the complexity and spatial heterogeneity of tumor-infiltrating lymphocytes in primary liver carcinoma. Oncotarget 2017;8(21):34844-57

78. Siegel RL, Miller KD, Jemal A. Cancer statistics, 2018. CA Cancer J Clin 2018;68(1):730

79. Skarke C, Lahens NF, Rhoades SD, Campbell A, Bittinger K, Bailey A, Hoffmann C, Olson RS, Chen L, Yang G, Price TS, Moore JH, Bushman FD, Greene CS, Grant GR, Weljie AM, FitzGerald GA. A Pilot Characterization of the Human Chronobiome. Sci Rep 2017;7(1):17141

80. Smuts JC, Holism and Evolution. Macmillan And Company Limited, 1927.

81. Sobradillo P, Pozo F, Agusti A. P4 medicine: the future around the corner. Archivos de bronconeumologia 2011;47:35-40

82. Soto AM, Sonnenschein C. Is systems biology a promising approach to resolve controversies in cancer research? Cancer cell international 2012;12:12

83. Soto AM, Longo G, Noble D. Preface to "From the century of the genome to the century of the organism: New theoretical approaches. Prog Biophys Mol Biol 2016;122(1):1-3

84. Stéphanou A, Volpert V. Hybrid Modelling in Biology: a Classification Review. Math Model Nat Phenom 2016;11:37-48

85. Thamrin C, Frey U, Kaminsky DA, Reddel HK, Seely AJ, Suki B, Sterk PJ. Systems Biology and Clinical Practice in Respiratory Medicine. The Twain Shall Meet. Am J Respir Crit Care Med 2016;194(9):1053-1061

86. van Kampen AH, Moerland PD. Taking Bioinformatics to Systems Medicine. Methods Mol Biol 2016;1386:17-41

87. Vogt H, Hofmann B, Getz L., The new holism: P4 systems medicine and the medicalization of health and life itself, Med Health Care Philos 2016;19(2):307-23

88. Williams EG, Wu Y, Jha P, Dubuis S, Blattmann P, Argmann CA, Houten SM, Amariuta T, Wolski W, Zamboni N, Aebersold R, Auwerx J. Systems proteomics of liver mitochondria function. Science 2016;352(6291):aad0189

89. Wolkenhauer O, Auffray C, Brass O, Clairambault J, Deutsch A, Drasdo D, Gervasio F, Preziosi L, Maini P, Marciniak-Czochra A, Kossow C, Kuepfer L, Rateitschak K, Ramis-Conde I, Ribba B, Schuppert A, Smallwood R, Stamatakos G, Winter F, Byrne H. Enabling multiscale modeling in systems medicine. Genome Med 2014;6(3):21

90. Yates LR, Seoane J, Le Tourneau C, Siu LL, Marais R, Michiels S, Soria JC, Campbell P, Normanno N, Scarpa A, Reis-Filho JS, Rodon J, Swanton C, Andre F. The European Society for Medical Oncology (ESMO) Precision Medicine Glossary. Ann Oncol 2017 